\title{
Doping dependence of the carrier lifetime crossover point upon dissociation of iron-boron pairs in crystalline silicon
}

\author{
D. Macdonald, ${ }^{\text {a) }}$ T. Roth, ${ }^{\text {b) }}$ and P. N. K. Deenapanray \\ Department of Engineering, College of Engineering and Computer Science, Australian National University, \\ Canberra, Australian Capital Territory 0200, Australia \\ T. Trupke and R. A. Bardos \\ Centre of Excellence for Advanced Silicon Photovoltaics and Photonics, University of New South Wales, \\ Sydney 2052, Australia
}

(Received 16 June 2006; accepted 10 August 2006; published online 4 October 2006)

\begin{abstract}
The excess carrier density at which the carrier lifetime in crystalline silicon remains unchanged after dissociating iron-boron pairs, known as the crossover point, is reported as a function of the boron dopant concentration. Modeling this doping dependence with the Shockley-Read-Hall model does not require knowledge of the iron concentration and suggests a possible refinement of reported values of the capture cross sections for electrons and holes of the acceptor level of iron-boron pairs. In addition, photoluminescence-based measurements were found to offer some distinct advantages over traditional photoconductance-based techniques in determining recombination parameters from low-injection carrier lifetimes. (C) 2006 American Institute of Physics. [DOI: 10.1063/1.2358126]
\end{abstract}

Iron is one of the most important metallic impurities in crystalline silicon materials made especially for solar cells, such as multicrystalline silicon. Whether present in pointlike form ${ }^{1}$ or as precipitates, ${ }^{2}$ its contribution to carrier recombination is often significant. In $p$-type silicon, which is most commonly used for photovoltaics, interstitial iron has a particularly strong recombination activity. ${ }^{3}$ Therefore, its unambiguous and rapid identification is of technological importance.

Recently, a fast and simple method for identifying interstitial Fe in boron-doped silicon has been proposed, via measurements of the so-called crossover point. ${ }^{4}$ This is defined as the excess carrier density $\Delta n$ at which the charge carrier lifetime remains unchanged when transforming the chemical state of nonprecipitated iron from $\mathrm{FeB}$ pairs to interstitial $\mathrm{Fe}$ $\left(\mathrm{Fe}_{i}\right)$. Such a transformation is easily achieved by strong illumination. ${ }^{5}$ Initial work ${ }^{4}$ indicated that the crossover point was almost independent of the boron dopant concentration $N_{A}$ (except when $N_{A}>5 \times 10^{16} \mathrm{~cm}^{-3}$ ), a feature which would have made identification of Fe in silicon even simpler. However, recent measurements on more lightly doped samples have clearly shown this to be incorrect. ${ }^{6}$ The purpose of this work is to accurately measure the position of this crossover point for a wide range of boron concentrations, in order to facilitate unambiguous identification of Fe contamination.

In principle, the crossover point can be predicted using the Shockley-Read-Hall model if the energy levels and capture cross sections for electrons and holes are known for both interstitial Fe and FeB pairs. ${ }^{7}$ However, there still exists considerable uncertainty in some of these values, particularly the capture cross sections. Consequently, we also infer from our measured crossover point data information about likely values of the capture cross sections.

Single-crystal silicon wafers with boron concentrations between $10^{14}$ and $2 \times 10^{16} \mathrm{~cm}^{-3}$ were etched, cleaned, and

\footnotetext{
${ }^{\text {a)} E l e c t r o n i c ~ m a i l: ~ d a n i e l . m a c d o n a l d @ a n u . e d u . a u ~}$

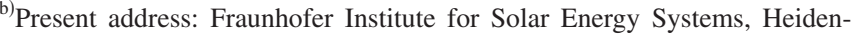
hofstr. 2, D-79110 Freiburg, Germany.
}

implanted with $70 \mathrm{keV} \mathrm{Fe} \mathrm{Fe}^{56}$ ions to a dose of $10^{11} \mathrm{~cm}^{-2}$ on one surface. A $30 \times 30 \mathrm{~mm}^{2}$ silicon aperture was used to define the implantations. After further cleaning, the samples were annealed at $900{ }^{\circ} \mathrm{C}$ in nitrogen for $1 \mathrm{~h}$, distributing the Fe approximately uniformly throughout the sample thickness. The resulting $\mathrm{Fe}$ concentrations are below the solid solubility limit at $900{ }^{\circ} \mathrm{C}$; hence precipitation is minimized. ${ }^{8}$ The low energy and dose used have been shown not to cause any residual damage after annealing that could otherwise affect the carrier lifetime. ${ }^{9}$ Surface passivation for sensitive bulk carrier lifetime measurements was achieved by plasmaenhanced chemical vapor deposited (PECVD) silicon nitride films deposited at $400{ }^{\circ} \mathrm{C}$.

Effective carrier lifetimes were measured primarily with the quasi-steady-state photoconductance (QSSPC) technique. ${ }^{10}$ This method allows a "scan" of the carrier lifetime as a function of the excess carrier concentration, an essential feature when measuring the position of the crossover point. Figure 1 shows an example of such a measurement, in which the sample has been measured before and after illumination to dissociate the FeB pairs, revealing the crossover point near $\Delta n=4 \times 10^{13} \mathrm{~cm}^{-3}$. The measurement temperature was $304 \pm 3 \mathrm{~K}$.

The QSSPC technique approximates the average excess

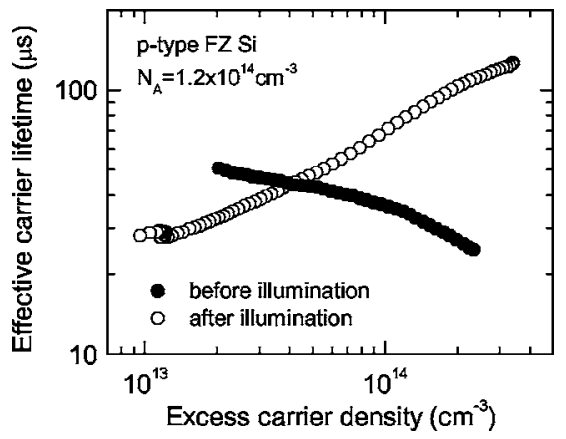

FIG. 1. QSSPC measurements of the excess carrier lifetime as a function of excess carrier density for an iron contaminated sample before and after light soaking. 


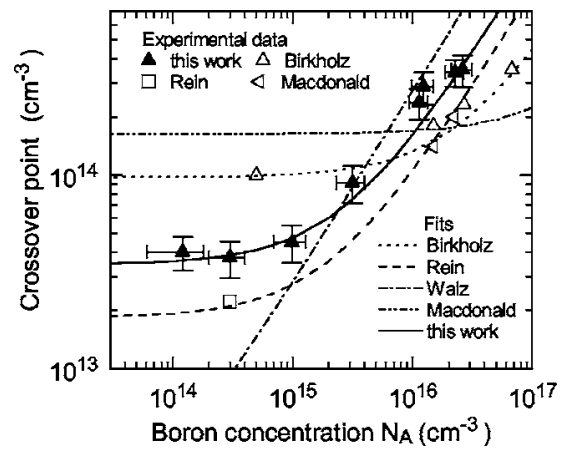

FIG. 2. Dependence of the crossover point on the boron dopant concentration. The fits are based on capture cross sections and energy levels either from the literature or as determined in this work.

carrier density as the total excess carrier density (determined from the photoconductance) divided by the sample thickness. This assumption becomes increasingly invalid when the minority carrier diffusion length $L_{D}$ becomes less than half the sample thickness. In such cases, we replaced the sample thickness with $2 L_{D}$ when calculating the average excess carrier density $\Delta n{ }^{11}$ This was necessary for the four most heavily doped samples. In calculating $L_{D}$, minority carrier diffusion coefficients were taken from Dorkel and Leturcq. ${ }^{12}$ In principle, lower Fe concentrations would avoid this problem; however, ion implantation doses below $10^{11} \mathrm{~cm}^{-2}$ are difficult to perform reliably with our implanter.

Figure 2 shows the measured values of the crossover point for various boron concentrations. Also shown are curves representing the expected crossover point based on energy levels and capture cross sections taken from the literature and are listed in Table I. ${ }^{6-8,13,14}$ Here we have used the expression for the crossover point derived by Birkholz et $a .^{7}$ All fits other than for Rein and Glunz assume the recombination parameters of Istratov et al. for $\mathrm{Fe}_{i}$. Where necessary we have used the thermal velocities of the authors, or $v_{\text {th }}=1.1 \times 10^{7} \mathrm{~cm} / \mathrm{s}$. ${ }^{15}$

The best fit to our data is given by the parameters recently determined by Rein and Glunz, ${ }^{6}$ which give values mostly lying within $50 \%$ of our measured data. This could be considered an acceptable uncertainty for most practical purposes. Nevertheless, it is worthwhile considering adjustments to the capture cross sections to give an even better fit. The question then is which cross sections should be adjusted? Both capture cross sections for $\mathrm{FeB}$ pairs are rather uncertain at present. For interstitial iron, on the other hand, the hole capture cross section is already well established, whereas the electron capture cross section is less certain, for example, those used by Rein and Glunz and by Istratov et al.

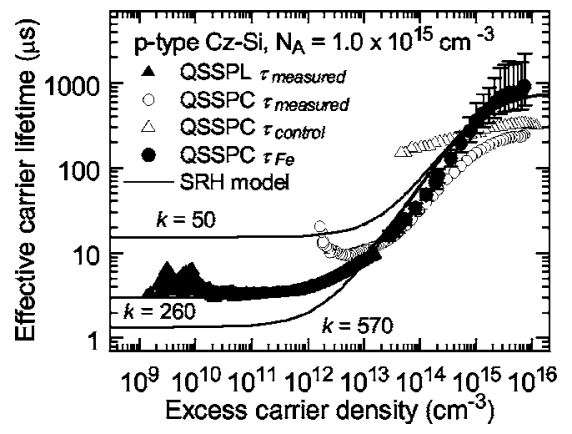

FIG. 3. Quasi-steady-state photoconductance (QSSPC) and photoluminescence (QSSPL) data measured on a boron-doped $14 \Omega \mathrm{cm}$ silicon wafer containing interstitial iron and a control wafer. Samples were light soaked prior to measurement to dissociate iron-boron pairs. Shockley-Read-Hall fits are shown for capture cross section ratios of $k=50,260$, and 570 .

are quite different. We therefore first attempted to independently determine an accurate value for the electron capture cross section for interstitial iron by means of true lowinjection lifetime measurements, as described below. Once this value is established, we are then able to adjust the capture cross sections for FeB pairs in order to obtain the best fit to our crossover point data.

In principle at least, measurement of the capture cross section ratio for interstitial iron should be relatively straightforward. According to the Shockley-Read-Hall model, for a deep level such as interstitial Fe, the true low-injection lifetime reflects the minority carrier capture cross section via the relation $\tau_{\text {low injection }}=1 /\left(v_{\text {th }} \sigma_{n}\left[\mathrm{Fe}_{i}\right]\right)$, where $v_{\text {th }}$ is the thermal velocity and $\left[\mathrm{Fe}_{i}\right]$ the interstitial $\mathrm{Fe}$ concentration. If the minority carrier capture cross section is much larger than the majority carrier capture cross section, as is the case for $\mathrm{Fe}_{i}$ in $p$-type silicon, then the high-injection lifetime is, conversely, limited by majority carrier capture. As a result, the ratio of the capture cross sections, $k=\sigma_{n}\left(\mathrm{Fe}_{i}\right) / \sigma_{p}\left(\mathrm{Fe}_{i}\right)$, can be determined directly from the ratio of the high-injection to lowinjection lifetimes. (Note that this approach is not valid for the shallower FeB pairs.) Given that the hole cross section for interstitial iron is well established, the electron cross section can then be directly determined.

With this in mind, low- and high-injection lifetimes were measured with a combination of quasi-steady-state photoluminescence (QSSPL), developed recently at UNSW, ${ }^{16}$ and QSSPC, as shown in Fig. 3. The sample was light soaked prior to measurement to break the FeB pairs. QSSPC is better suited to high-injection measurements due to the ability to use strong white light to inject many carriers. On the other hand, QSSPL is suited to low-injection measurements, since

TABLE I. Reported energy levels and capture cross sections for interstitial iron $\left(\mathrm{Fe}_{i}\right)$ and the acceptor level of FeB pairs.

\begin{tabular}{llcccl}
\hline \hline & $E_{T}(\mathrm{eV})$ & $\sigma_{n}\left(\mathrm{~cm}^{2}\right)$ & \multicolumn{1}{c}{$\sigma_{p}\left(\mathrm{~cm}^{2}\right)$} & $k=\sigma_{n} / \sigma_{p}$ & \multicolumn{1}{c}{ Reference } \\
\hline $\mathrm{Fe}_{i}$ & $E_{V}+0.38$ & $4 \times 10^{-14}$ & \multicolumn{1}{c}{$7 \times 10^{-17}$} & 570 & \multicolumn{1}{c}{ Istratov et al., 1999 (Ref. 8) } \\
& $E_{V}+0.39$ & $3.6 \times 10^{-15}$ & $7 \times 10^{-17}$ & 51 & Rein and Glunz, 2005 (Ref. 6) \\
$\mathrm{FeB}$ & $E_{C}-0.29$ & $2.5 \times 10^{-15}$ & $3 \times 10^{-14}$ & 0.08 & Walz et al., 1996 (Ref. 13) \\
& $E_{C}-0.23$ & $3 \times 10^{-14}$ & $2 \times 10^{-15}$ & 15 & Macdonald et al., 2001 (Ref. 14) \\
& $E_{C}-0.26$ & $1.4 \times 10^{-14}$ & $1.1 \times 10^{-15}$ & 13 & Birkholz et al., 2005 (Ref. 7) \\
& $E_{C}-0.26$ & $2.5 \times 10^{-15}$ & $5.5 \times 10^{-15}$ & 0.45 & Rein and Glunz, 2005 (Ref. 6) \\
& $E_{C}-0.26$ & $5 \times 10^{-15}$ & $3 \times 10^{-15}$ & 1.7 & This work \\
\hline
\end{tabular}


it is immune to measurement artifacts caused by depletion region modulation ${ }^{16}$ (DRM) or minority carrier trapping. ${ }^{17}$ Figure 3 illustrates this point. The QSSPL data show that the true recombination lifetime continues to decrease to values well below the minimum in the QSSPC data. The sudden increase in the measured lifetime for the QSSPC data below $\Delta n=10^{13} \mathrm{~cm}^{-3}$ is apparently associated with quasistatic variation of the depletion region width caused by fixed charges present in the silicon nitride film. ${ }^{18}$

The low-injection lifetime from the QSSPL data was found to be $3.2 \mu \mathrm{s}$. The high-injection lifetime is more difficult to measure accurately, because the imperfect passivation of the surfaces by the SiN films causes surface recombination to impact on the measurements in this range. However, the lifetime due to $\mathrm{Fe}_{i}, \tau_{\mathrm{Fe}}$, can be estimated via $1 / \tau_{\mathrm{Fe}}=1 \tau_{\text {measured }}-1 / \tau_{\text {control }}$, and is shown in Fig. 3 . Since the control lifetime is close to the lifetime measured in the Feimplanted sample, the corresponding high-injection lifetime for $\mathrm{Fe}_{i}$ can only be estimated as lying within the range of $500-2000 \mu \mathrm{s}$, with the most likely value near $800 \mu \mathrm{s}$ (assuming $10 \%$ uncertainty in the lifetime measurements). This corresponds to a $k$ value between 160 and 630, with the most likely value near 260 . This range is consistent with the value of Istratov et al. in Table I, while being outside that of Rein and Glunz. The result is also consistent with our previous work, which indicated a value of $k>75 .{ }^{3}$

Figure 3 also shows Shockley-Read-Hall fits for $\mathrm{Fe}_{i}$ using both $k=50$ (as proposed by Rein and Glunz), $k=570$ (Istratov et al.), and $k=260$ (as determined above). The $\mathrm{Fe}_{i}$ concentration was chosen to be $1.7 \times 10^{12} \mathrm{~cm}^{-3}$ ( $85 \%$ of the value expected from the implant dose-it is typical to lose a small fraction to precipitation at the implanted surface). The hole capture cross section for $\mathrm{Fe}_{i}$ was taken as the wellestablished value of $7 \times 10^{-17} \mathrm{~cm}^{2}$, and the fact that it yields a reasonable lifetime value at high injection indicates that it is accurate. Our own value of $k=260$ fits the injectiondependent data best and implies an electron capture cross section for electrons of $2 \times 10^{-14} \mathrm{~cm}^{2}$-half that of Istratov et al. However, considering the uncertainties in the highinjection data, the value of Istratov et al. is also reasonable. In addition, a slight amount of FeB re-pairing prior to our QSSPL measurement could have occurred, which would act to reduce the apparent value of $k$. In any case, it turns out that choosing either of these values makes little difference to the calculation of either the crossover point or the interstitial Fe concentration (provided that the lifetime measurements before and after $\mathrm{FeB}$ dissociation are performed above the crossover point). As such, we propose to retain the value of Istratov et al. for the electron capture cross section for interstitial iron.

Returning to the doping dependence of the crossover point in Fig. 2, we find that the best values for the FeB cross sections were $\sigma_{n}(\mathrm{FeB})=(5 \pm 1.5) \times 10^{-15} \mathrm{~cm}^{2}$ and $\sigma_{p}(\mathrm{FeB})$ $=(3 \pm 1) \times 10^{-15} \mathrm{~cm}^{2}$. These values, in tandem with those of Istratov et al. for interstitial iron, are therefore recommended for calculations of the crossover point or the interstitial $\mathrm{Fe}$ concentration.

A possible explanation for the discrepancy between the $k$ values determined here (and by Istratov et al.) and those found using photoconductance-based lifetime techniques is that the latter may be affected by DRM or minority carrier trapping. Such effects are particularly important for defects with highly asymmetrical cross sections, such as $\mathrm{Fe}_{i}$, since the true low-injection lifetime occurs at lower excess carrier densities than, for example, $\mathrm{FeB}$ pairs. For $\mathrm{Fe}_{i}$ concentrations around $10^{12} \mathrm{~cm}^{-3}$, trapping effects caused by the $\mathrm{Fe}_{i}$ centers themselves can affect the low-injection lifetime measurements at room temperature. ${ }^{19,20}$ Note that such "selftrapping" does not lead to a characteristic increase in the apparent lifetime at low-injection levels, as occurs for trapping through other types of defects, but rather an injectionindependent lifetime that is higher than the true low-injection lifetime. ${ }^{19,20}$ This makes such self-trapping difficult to identify. However, it is also true that trapping effects would rapidly diminish at higher measurement temperatures (as used in temperature-dependent lifetime spectroscopy). On the other hand, DRM artifacts may persist at higher temperatures.

In summary, we have measured the doping dependence of the $\mathrm{Fe}_{i} / \mathrm{FeB}$ crossover point in crystalline silicon. This should aid fast identification of Fe contamination in device processing, especially for solar cells. The doping dependence of the crossover point also suggests slight modifications to the best existing values of the capture cross sections for electrons and holes of the acceptor level of FeB pairs. Finally, due to their immunity to measurement artifacts occurring at low injection, photoluminescence measurements appear to offer some advantages over the traditional photoconductance methods often used for lifetime spectroscopy.

This work has been supported by the Australian Research Council. R. Elliman and C. Jagadish of the Research School of Physical Sciences and Engineering, ANU, kindly permitted access to the ion implanter and PECVD reactor.

${ }^{1}$ R. A. Sinton, T. Mankad, S. Bowden, and N. Enjalbert, Proceedings of the 19th European Photovoltaic Solar Energy Conference, Paris, France (WIP, Munich, 2004), p. 520.

${ }^{2}$ T. Buonassisi, A. A. Istratov, M. Heuer, M. A. Marcus, R. Jonczyk, J. Isenberg, B. Lai, Z. Cai, S. Heald, W. Warta, R. Schindler, G. Willeke, and E. R. Weber, J. Appl. Phys. 97, 074901 (2005).

${ }^{3}$ D. Macdonald and L. J. Geerligs, Appl. Phys. Lett. 85, 4061 (2004).

${ }^{4}$ D. Macdonald, L. J. Geerligs, and A. Azzizi, J. Appl. Phys. 95, 1021 (2004).

${ }^{5}$ G. Zoth and W. Bergholz, J. Appl. Phys. 67, 6764 (1990).

${ }^{6}$ S. Rein and S. W. Glunz, J. Appl. Phys. 98, 113711 (2005).

${ }^{7}$ J. E. Birkholz, K. Bothe, D. Macdonald, and J. Schmidt, J. Appl. Phys. 97, 103708 (2005).

${ }^{8}$ A. A. Istratov, H. Hieslmair, and E. R. Weber, Appl. Phys. A: Mater. Sci. Process. 69, 13 (1999).

${ }^{9}$ D. Macdonald, P. N. K. Deenapanray, and S. Diez, J. Appl. Phys. 96, 3687 (2004).

${ }^{10}$ R. A. Sinton and A. Cuevas, Appl. Phys. Lett. 69, 2510 (1996).

${ }^{11}$ S. Bowden and R. A. Sinton, 14th Workshop on Crystalline Silicon Solar Cells and Modules, Winter Park, CO (NREL, Golden, CO, 2004), p. 212.

${ }^{12}$ J. M. Dorkel and P. Leturcq, Solid-State Electron. 24, 821 (1981).

${ }^{13}$ D. Walz, J.-P. Poly, and G. Kamarinos, Appl. Phys. A: Mater. Sci. Process. 62, 345 (1996).

${ }^{14}$ D. Macdonald, A. Cuevas, and J. Wong-Leung, J. Appl. Phys. 89, 7932 (2001).

${ }^{15}$ W. M. Bullis and H. R. Huff, J. Electrochem. Soc. 143, 1399 (1996).

${ }^{16} \mathrm{~T}$. Trupke and R. Bardos, Proceedings of the 31st IEEE Photovoltaic Specialists Conference, Orlando, FL (IEEE, New York, 2005), p. 903.

${ }^{17}$ R. A. Bardos, T. Trupke, M. C. Schubert, and T. Roth, Appl. Phys. Lett. 88, 053504 (2006).

${ }^{18}$ P. J. Cousins, D. H. Neuhaus, and J. E. Cotter, Appl. Phys. Lett. 95, 1854 (2004).

${ }^{19}$ J. S. Blakemore, Semiconductor Statistics, International Series of Monographs on Semiconductors Vol. 3 (Pergamon, Oxford, 1962).

${ }^{20}$ D. Macdonald and A. Cuevas, Phys. Rev. B 67, 075203 (2003). 